\title{
Isolation and quarantine during pandemic (H1N1) 2009 influenza in NSW: the operational experience of public health units
}

\section{Philippa L. Binns ${ }^{\mathrm{A}, \mathrm{D}}$, Vicky Sheppeard ${ }^{\mathrm{B}}$ and Michael P. Staff ${ }^{\mathrm{C}}$}

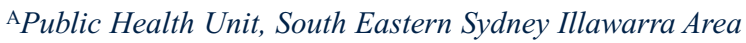
Health Service

${ }^{\text {B }}$ Public Health Unit, Sydney West Area Health Service

${ }^{\mathrm{C}}$ Public Health Unit, Northern Sydney Central Coast Area Health Service

${ }^{\mathrm{D} C o r r e s p o n d i n g ~ a u t h o r . E m a i l: ~ P h i l i p p a . B i n n s @ s e s i a h s . ~}$ health.nsw.gov.au
The Australian Health Management Plan for Pandemic Influenza identifies discrete stages of an Australian response to pandemic influenza, including DELAY and CONTAIN phases. ${ }^{1}$ The objective of the DELAY phase is to prevent or slow the entry of the virus into Australia using border measures and increased vigilance for cases.
Once the pandemic virus has entered Australia, but has caused only a small number of cases, measures outlined under the CONTAIN phase are designed to prevent local community transmission becoming established.

The NSW Human Influenza Pandemic Plan ${ }^{2}$ identifies a specific role for public health authorities in combating the threat of a novel influenza virus such as pandemic (H1N1) 2009 influenza. New South Wales (NSW) has a welldeveloped network of public health units (PHUs) located within its eight area health services (AHSs) and in Justice Health. Four PHUs are classified as metropolitan (Northern Sydney Central Coast, South Eastern Sydney Illawarra, Sydney South West and Sydney West) and four as rural (Greater Southern, Greater Western, Hunter New England and North Coast) based on the predominant nature of the geographical area that they serve. The workforce in each PHU is made up of a varying combination of senior public health managers and physicians, medical officers, public health nurses, surveillance officers, epidemiologists, environmental health officers, immunisation practitioners and administration officers dependent upon local population factors and AHS need. Each PHU, and the network it comprises, maintains links with the NSW Department of Health while maintaining responsibility for managing public health issues within the geographical region covered by its AHS.

To guide a consistent national public health response during the pandemic, the Communicable Diseases Network Australia (CDNA) published a series of Interim Guidelines for Public Health Units. ${ }^{3}$ To achieve the objectives of the DELAY and CONTAIN phases, rapid identification and isolation of potential cases, as well as specimen collection for laboratory testing, needed to be co-ordinated by PHUs. People in close contact with a suspected case with an Influenza A positive test, or probable or confirmed cases, also required public health interventions including quarantine and chemoprophylaxis where indicated.

This paper describes the operational experiences of PHUs in NSW during the early phases of the pandemic H1N1 (2009) influenza (illustrated by three case studies) and highlights the lessons learned. 


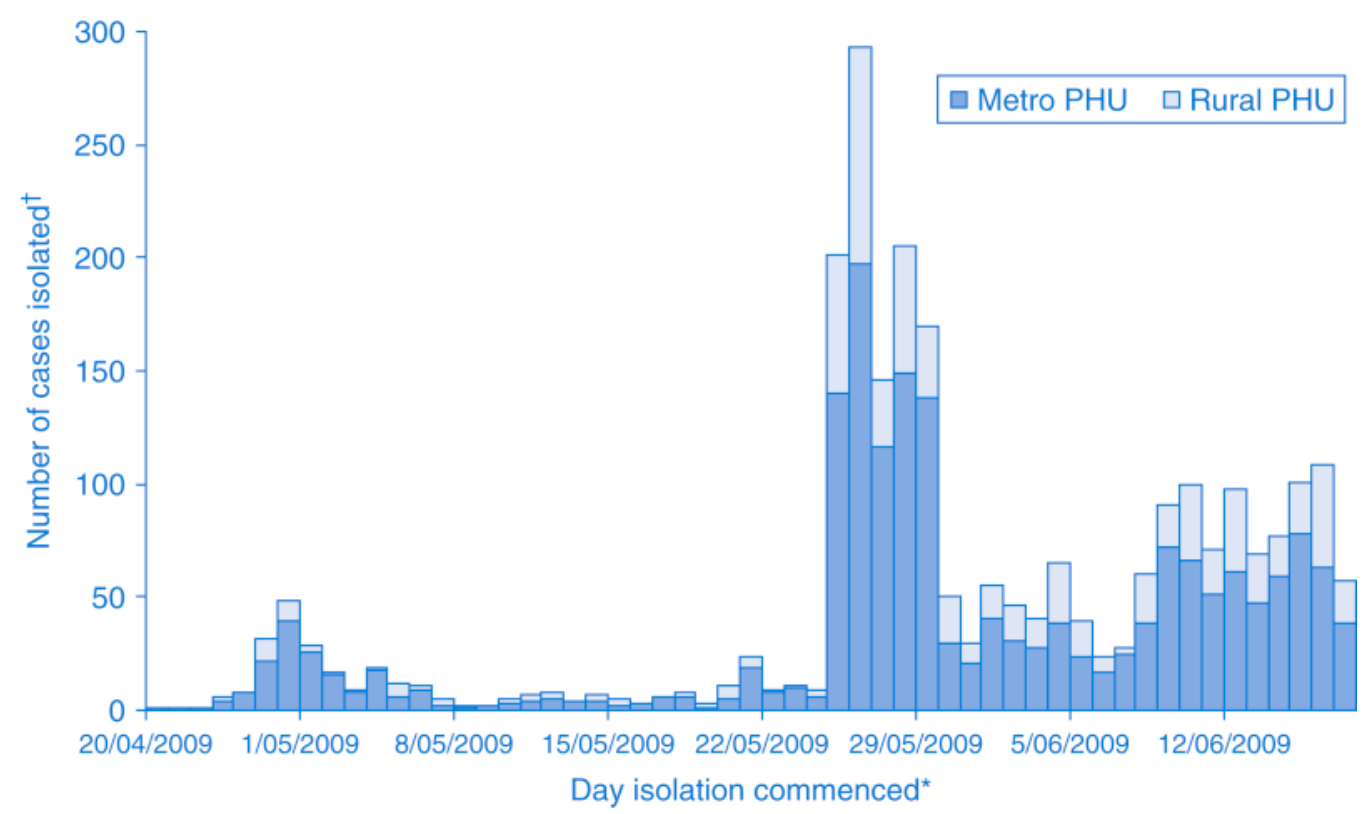

Figure 1. Number of cases notified in NSW requiring isolation during pandemic (H1N1) 2009 influenza DELAY and CONTAIN phases managed by NSW public health units (PHU).

*Date is reported as the recorded date of commencement of isolation, or if missing, the date of isolation follow-up or release, whichever is earlier.

tThe number of cases refers to any case investigated and recorded as being placed in home isolation, or if missing, those that have a daily follow-up record, duration of home isolation $>0$ days, record of completing isolation or release date documented. Source: NSW Health swine flu surveillance and outbreak database, NetEpi.

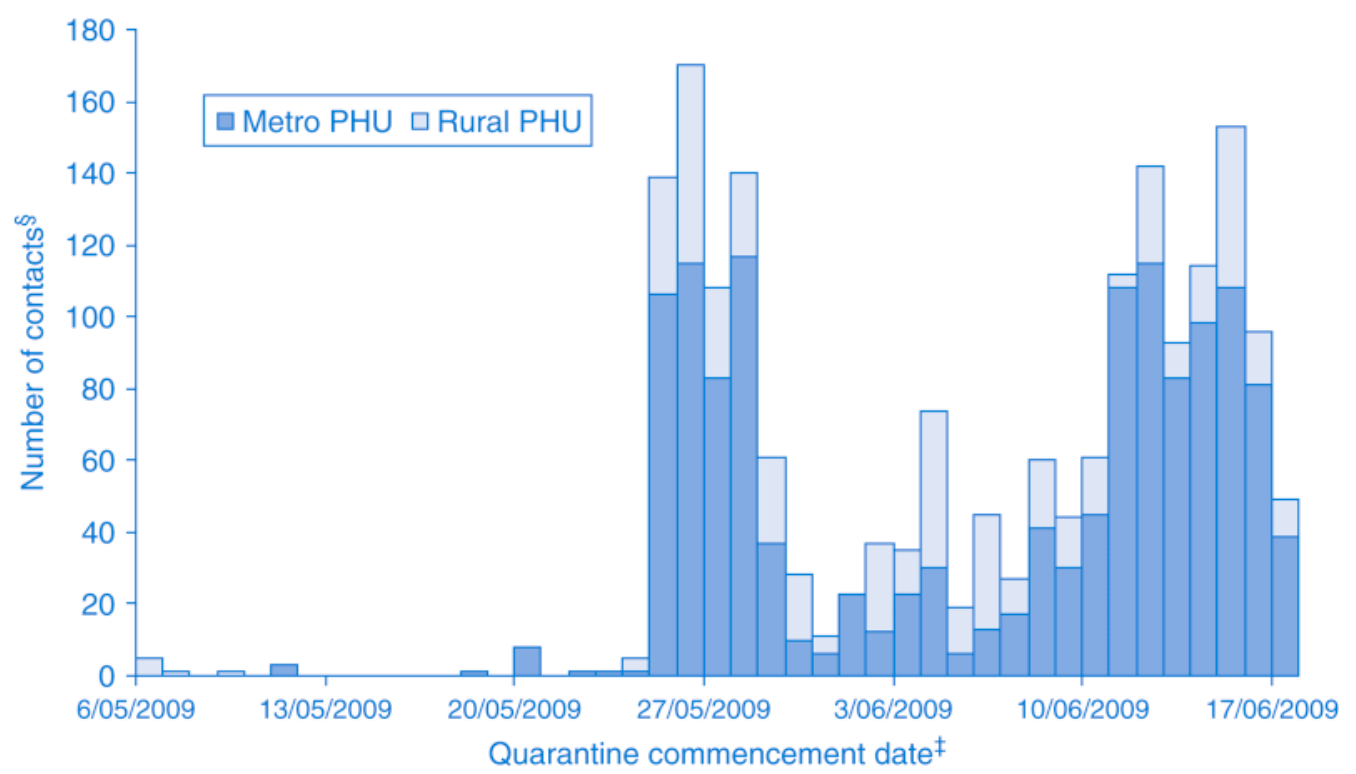

Figure 2. Number of contacts ${ }^{\S}$ in NSW requiring quarantine during pandemic (H1N1) 2009 influenza DELAY and CONTAIN phases managed by NSW public health units.

FDate is reported as the recorded date of commencement of quarantine, or if this date is missing, the date of first quarantine follow-up or release, whichever is earlier.

${ }^{\S}$ The number of contacts refers any contact investigated and recorded as being placed in home quarantine, or if data missing, those that have consented to quarantine, have a daily follow-up record, duration of quarantine $>0$ days, record of completing quarantine or release date documented.

Source: NSW Health swine flu surveillance and outbreak database, NetEpi. 


\section{Methods}

Data for case notifications and contacts managed by PHUs during the DELAY and CONTAIN phases were extracted on 16 October 2009 from NetEpi, a secure web-based multi-user access surveillance tool provided by NSW Health. ${ }^{4}$ Due to different standards of data recording across PHUs, individuals in isolation/quarantine were defined as: any case/contact investigated and recorded as being placed in home isolation/quarantine, or if these data were missing, a commencement date, daily follow-up, duration of home isolation/quarantine greater than 0 days, isolation/quarantine completion or release date documented. Commencement date is reported as: the recorded date of commencement of isolation/quarantine, or if these data are missing, the date of follow-up or release, whichever is earlier. Stata ${ }^{\circledR 5}$ was used for analyses and Microsoft ${ }^{\circledR}$ Office Excel for graphs.

The description of PHU operational experience is based both on our own personal experiences and reflections, and also on observations and suggestions from the public health network as discussed in a variety forums such as teleconferences, debriefs, meetings and informal discussions since the beginning of the pandemic. In response to an email request from one of the authors, PHUs provided information about the numbers of courses of medication and isolation packs distributed during DELAY and CONTAIN phases.

\section{Cases and contacts recorded during DELAY and CONTAIN phases}

Until the declaration of the PROTECT phase on 17 June, 3070 notifications of potential cases were investigated and managed by NSW PHUs. Of these, 376 were confirmed as cases, 53 probable cases and 93 were suspected cases (unable to confirm/exclude), as defined by the CDNA guidelines. ${ }^{3}$ Follow up of the 376 confirmed cases and the 53 probable cases during the CONTAIN phase, as recommended by the CDNA guidelines, identified 1894 contacts that were managed by PHUs. The majority of these cases and contacts were managed by the four metropolitan PHUs. There were three distinct peaks in the number of people requiring isolation (Figure 1) and two peaks for those requiring quarantine (Figure 2). The highest peak corresponded to the week following disembarkation of passengers from the Pacific Dawn cruise ship (Case study 1).

\section{Public health unit response}

PHUs were first notified of the outbreak of pandemic (H1N1) 2009 influenza on 25 April and required a rapid re-orientation and escalation of their activity to implement the functions associated with the DELAY phase. While PHUs took different approaches, the Incident Control System was adopted by most to adapt rapidly to the new situation, and proved to be an efficient and effective management structure. The Incident Control System applied in a PHU setting allows flexibility in the personnel assigned to roles. In most instances, however, the PHU Director was the public health controller; public health nurses and surveillance officers were operations officers; epidemiologists were planning officers; and environmental health officers and administrative staff were logistics officers.

\section{Staffing}

Surge staff were required for all functions. To supplement the PHU planning teams, epidemiologists were sourced from within AHS Population Health Divisions. Generally, logistics functions were undertaken by Population Health Division staff who were moved from their normal duties. Operations, including surveillance, follow-up and answering inquiries, required the largest surge capacity. This was accommodated by co-opting nurses and other health professionals mainly sourced from immunisation programs, community health services and population health divisions.

The training of staff to undertake the operations role independently took considerable time, thus it was important that surge staff could be assigned to pandemic influenza operations for at least one week. The online Pandemic Influenza Program ${ }^{7}$ developed by Hunter New England Area Health Service and the NetEpi Training developed by NSW Health provided useful orientation and training for new operations staff. Job action sheets and standard operating procedures were also developed by planning sections, and surge staff worked closely with experienced surveillance officers during their first few days in operations.

\section{Communications}

During the DELAY and CONTAIN phases, communication was a critical and complex task. Case definitions and operational requirements changed frequently, which affected PHU staff, primary health care providers and the general public. PHUs were seen as a source of definitive information resulting in a significant escalation of incoming calls. Daily staff briefings or other mechanisms were implemented to ensure that all staff were aware of current policies and procedures. Some PHU telephone systems were overwhelmed and incoming calls had to be diverted to a central Department of Health call centre to be triaged.

\section{Information tools}

Information exchange between the Department of Health and the network occurred via email, a wiki (a web-based tool with administrative functions allowing collaboration, and information sharing for those who have access ${ }^{8}$ ) and daily teleconferences. To a large extent the wiki and email fulfilled a similar role. Over time it became difficult to find specific information on the wiki, so more reliance was placed on email or the daily teleconference. However, 
Case study 1. Preventing widespread community transmission of pandemic (H1N1) influenza from confirmed cases on a cruise ship

After passengers and crew disembarked from the Pacific Dawn cruise ship in Sydney on 25 May, pandemic (H1N1) 2009 influenza infections were confirmed in a small number of passengers, despite the ship not visiting areas known to be affected by the pandemic. ${ }^{6}$ To prevent widespread community transmission, PHUs - in collaboration with NSW Department of Health staff - needed immediate surge capacity to: contact those who had reported illness on their Health Declaration Cards, manage those requiring isolation or quarantine, and respond to the increase in telephone calls from the public and health professionals. Within a week, PHUs investigated and managed 1095 case and 662 contact notifications; a third of all notifications for the entire DELAY and CONTAIN period. Of these, 1095 individuals were managed in isolation by PHUs and 657 in quarantine (Table 1). Given that daily follow-up was also required, efficient use of resources was essential and usual work patterns were adjusted to cope with this sudden escalation in workload. Clear characterisations of workflows (Figure 3) assisted teams, especially while rapidly orientating the surge staff in their roles. The co-ordinated response across the country and within NSW appeared to contain the effect on the broader community.

\section{Case study 2. An outbreak of pandemic (H1N1) in a boarding school in metropolitan Sydney}

Five of the 11 secondary school boarding colleges in the northern region of metropolitan Sydney had outbreaks of laboratory-confirmed pandemic (H1N1) 2009 influenza during the period 23 June-30 June. The estimated attack rates in colleges ranged from 10 to $30 \%$ with approximately 320 cases occurring in total. Most colleges also reported concurrent illness among non-boarders and although most boarders experienced relatively mild illness, the outbreaks caused substantial disruption. One college of 650 boarders was forced to close on 26 June (4 days early for the winter school holidays) as the college felt it could not care for its students adequately. Simple respiratory hygiene measures and restrictions on student movement were employed in an attempt to control the outbreaks. Antiviral use for prophylaxis and/or treatment was reserved for students 'at risk' of influenza complications. The return to school on 27 July following the 3-week holiday break did not see a recurrence of illness in the colleges.

\section{Case study 3. An outbreak of pandemic (H1N1) influenza in a Pacific Islander community}

In early June, the PHU, Sydney South West became aware of pandemic (H1N1) 2009 influenza in a Pacific Islander community after a family of four presented to a local hospital flu clinic. Contact tracing revealed the index case to be a relative who had returned from Canberra by car 4 days earlier. Nine family members and the three other passengers in the car were subsequently diagnosed with pandemic (H1N1) 2009 influenza. In addition to family and social events, the index case reported attending a community conference with 700 participants from around Australia.

Efforts by the PHU to identify, assess and isolate contacts were complicated by several factors including there being: independent family groups within extended households; difficulty in identifying family leaders; and variable levels of transfer of information within and between groups. These barriers were overcome with the assistance of the Conference President, who disseminated an alert to community leaders via email on behalf of the PHU. One result from this action was 20 community members presenting for assessment at a hospital flu clinic. Regrettably, the majority had been symptomatic for too long to benefit from antiviral treatment.

problems arose when email systems became overloaded, with some PHUs experiencing significant delays in receiving new documents and protocols.

The NetEpi tool facilitated communication about cases between laboratories, PHUs and the Department of Health. The ability to access NetEpi independently of the NSW Health computer network was compatible with operations that extended almost around the clock for several weeks, allowing operations to continue from home or when computer networks were down (provided remote broadband facilities were available). In most instances, results were entered at the laboratory, allowing PHU staff to provide them to cases and contacts under investigation more rapidly than relying on faxes or telephone calls from the laboratory. NetEpi was invaluable in providing a central repository of data about the numbers of cases and contacts and their status, enabling the public health network near real-time information to assist in their management and to monitor the evolution of the pandemic in NSW. Some limitations included the availability of paper forms for operations staff that were current with the rapidly changing data collection fields and protocols, and the absence of data entry conventions and a manual to assist users to generate reports. PHUs sought more responsiveness in making changes to the data collection fields to accommodate the rapid change in case definitions and protocols that occurred. The extraordinary demands on the 
Table 1. Number of people in NSW requiring isolation or quarantine, and managed by public health units (PHU) during the peak week of containment activity

\begin{tabular}{|c|c|c|c|c|}
\hline $\begin{array}{l}\text { Notifications requiring isolation } \\
\text { or quarantine }\end{array}$ & $\begin{array}{l}\text { Metropolitan } \\
\text { PHU }\end{array}$ & $\begin{array}{l}\text { Rural } \\
\text { PHU }\end{array}$ & $\begin{array}{l}\text { Other/ } \\
\text { Missingll }\end{array}$ & Total \\
\hline Confirmed cases & 49 & 16 & 2 & 67 \\
\hline Other case" & 742 & 287 & 142 & 1171 \\
\hline Total cases in isolation & 791 & 304 & 144 & 1239 \\
\hline Total contacts in quarantine & 474 & 183 & 91 & 748 \\
\hline Total managed in isolation and quarantine & 1265 & 487 & 235 & 1987 \\
\hline
\end{tabular}

system highlighted the need to invest in surge capacity for staff with information technology skills to support frontline operational staff.

\section{Logistics}

PHUs adopted a number of different mechanisms to facilitate specimen collection and testing. Suspected cases were encouraged to attend hospital emergency departments rather than general practice for assessment and treatment, however a number of people fitting the case definition did not have swabs taken in either setting. In some units, public health nurses were trained to collect nose and throat swabs safely and conducted home visits to obtain specimens from all affected family members. Other units organised for taxis to transport suspected cases to emergency departments so that swabs could be obtained.

Distribution of antiviral medication and home isolation/quarantine packs to cases and contacts was identified as a function for PHUs in the NSW Health Interim Influenza Pandemic Action Plan, ${ }^{9}$ and this was the first time such an activity was undertaken on a large scale. Households requiring medication and packs were identified by surveillance officers during telephone interviews. Medication was then prescribed by a medical officer or registered nurse under a standing order. The logistics team co-ordinated home deliveries either by logistics officers or commercial couriers. The four metropolitan PHUs reported that they distributed approximately 900 courses of antiviral medication during this period. Of the four PHUs that had records available, 470 isolation/quarantine packs containing gloves, masks, alcohol-based cleaning agent, tissues and information were distributed to households in conjunction with the antiviral medications. Packs were also provided to hospital emergency departments to be given to cases when they were discharged.

Where cases occurred in institutional settings such as schools or residential care facilities it was often more efficient for PHUs to conduct a clinic onsite so that information could be provided to the entire group at one time, and medication dispensed directly to cases and contacts or to their carers. One such clinic is described in the article by Weston and Truman in this issue.

\section{Conclusions and lessons learned}

After years of planning for an influenza pandemic, the lessons learned from this response will be important to incorporate when these plans are revised. These are:

- A web-based multi-user access database with both reporting and case management capacity is essential. NetEpi has the capacity to fulfil this role. The number of staff with the capability to manage and adapt such outbreak tools should be increased, perhaps through developing skills across the network.

- The Incident Control System works well if implemented in a flexible manner. Incorporating Incident Control System training into professional development for all new and existing staff would improve familiarity and ease in adapting this system to public health emergency responses in the future.

- The role of Surveillance Officer/Public Health Nurse poses the greatest challenge when considering workforce surge. The skills needed are specialised and require considerable training. Negotiation with managers of identified surge staff is paramount to ensure capacity for an available and trained workforce to respond to public health emergencies, as is supporting staff to rapidly adapt to different and unfamiliar work patterns.

- Effective network communication is a critical element of emergency response. Clear communication channels are important. Use of several mechanisms (email, wiki and teleconference) increases reliability but can result in redundancy and information overload. The network should develop familiarity with using wikis as communication tools and contribute to the evolution of a more robust version. 


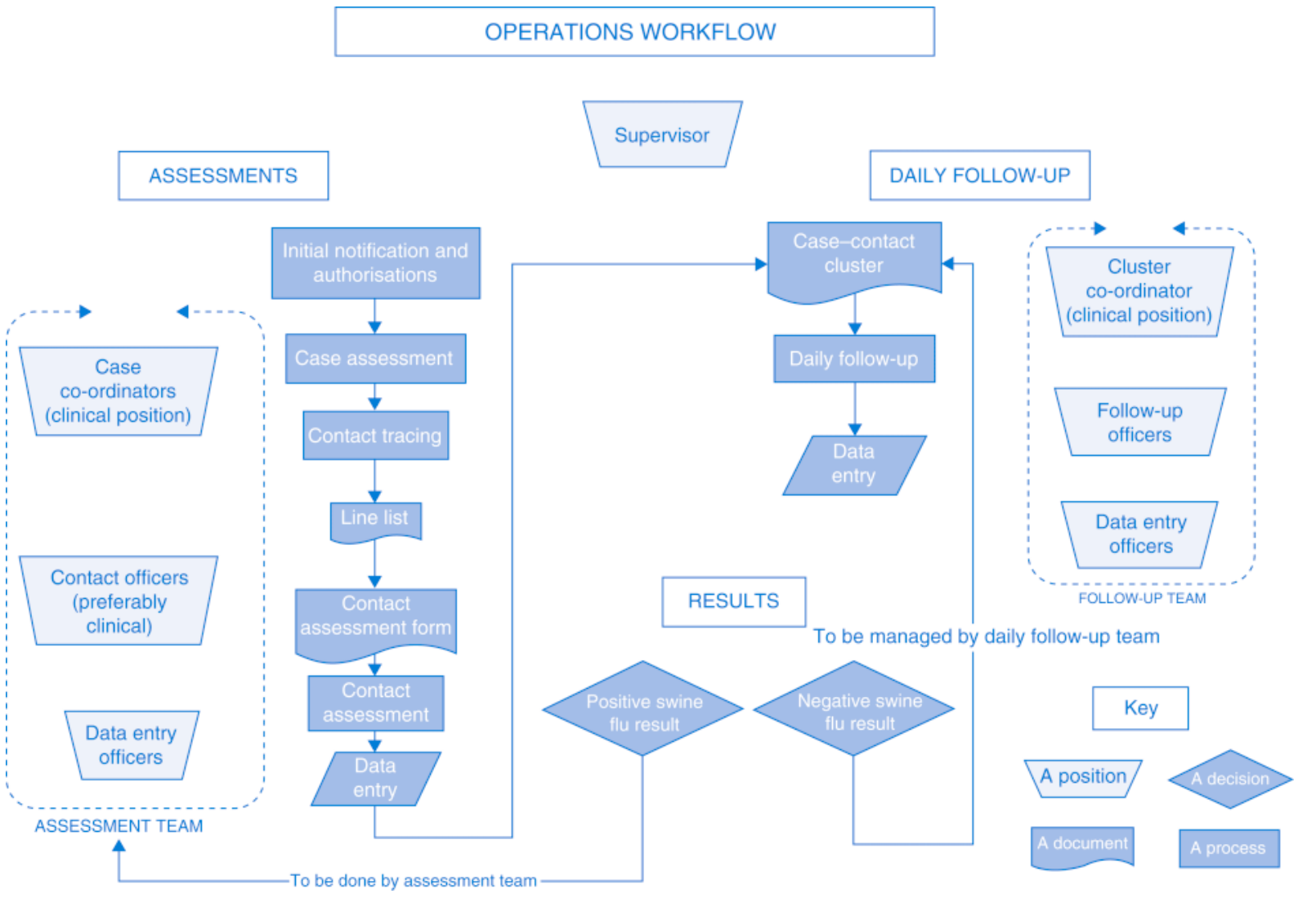

Figure 3. An example of a public health unit operations section workflow during DELAY and CONTAIN phases of the pandemic (H1N1) 2009 influenza response in NSW.

\section{Acknowledgments}

The authors wish to acknowledge all area health service staff who contributed to the control of the pandemic. We would like to thank Victoria Bowden, Patrick Maywood and Caroline Turnour of the Public Health Unit, Sydney South West Area Health Service for contributing their experience of an outbreak in the Pacific Islander community. We would also like to acknowledge the Department of Health team for their leadership during the pandemic.

\section{References}

1. Office of Health Protection, Department of Health and Ageing, Commonwealth of Australia. Australian Health Management Plan for Pandemic Influenza. 2008. Available from: http://www.flupandemic.gov.au/internet/panflu/publishing.nsf/ Content/ahmppi (Cited 1 October 2009.)

2. Influenza Pandemic Taskforce NSW. NSW Human Influenza Pandemic Plan. Available from: http://www.emergency.nsw. gov.au/content.php/583.html (Cited 1 October 2009.)

3. Communicable Diseases Network Australia. H1N1 influenza 09 (Human Swine Influenza) Infection 'Contain Phase' Interim Guidelines for Australian Public Health Units. 2009.
4. NetEpi Case Manager. Available from: http://netepi.sourceforge.net

5. StataCorp. Stata Statistical Software: Release 11. College Station, TX: StataCorp LP 2009.

6. NSW Department of Health. Communicable Diseases Report, NSW, May and June 2009. N S W Pub Health Bull 2009; 20(7-8): 133-5.

7. Hunter New England Area Health Service Population Health and Organisational Capability and Learning Unit. Pandemic Influenza Program. Available from: http://mylink. hnehealth.nsw.gov.au/course/view.php?id=427 (Cited 19 October 2009.)

8. Hannover Fairs. Wiki for Government. 2009. Available from: http://www.gov2.com.au/wiki-for-government (Cited 9 November 2009.)

9. NSW Department of Health. NSW Health Interim Influenza Pandemic Action Plan. 2005. Available from: http://www. health.nsw.gov.au/pubs/2005/pdf/pandemic_ap.pdf (Cited 20 October 2009.) 